\title{
The role of phase transitions in the evolution of dispersion parti- cles in chromium bronzes upon the equal channel angular pressing
}

\author{
I.A. Faizov ${ }^{1}$, G.I. Raab ${ }^{1}$, S.N. Faizova ${ }^{1, \dagger}$, N.G. Zaripov ${ }^{1}$, D.A. Aksenov ${ }^{2}$ \\ †snfaiz@mail.ru \\ ${ }^{1}$ Ufa State Aviation Technical University, 12 K. Marks St., Ufa, 450000, Russia \\ ${ }^{2}$ Institute of Molecule and Crystal Physics Ufa Research Center of RAS, 71 pr. Oktyabrya, Ufa, 450000, Russia
}

\begin{abstract}
Samples of a diluted dispersion-strengthening alloy of the $\mathrm{Cu}$-Cr-Zr system were processed with a combination of some severe plastic deformation methods. Preliminary, the samples were quenched into water from a pre-melting temperature to create a supersaturated solid solution concentration. In the course of the experiment the average sizes and distribution density of particles with different chemical compositions undergo an evolution that indicates that phase transformations occur even during the cold deformation. A decomposition of the solid solution due to the deformation-induced acceleration of diffusion certainly contributes to this evolution, but the whole complex of the observed results cannot be explained only with this relatively well-studied phenomenon and implies the presence of the deformation-induced dissolution of the second-phase particles. A possible mechanism of the dissolution linking this process to a mechanical fragmentation of the particles in the course of severe plastic deformation is discussed. A dependence of the dissolution efficiency on the composition and related morphology of the particles was also noted. A conclusion on the simultaneously occurring of the kinetically opposite phase transitions is also confirmed by an observation of changes in the lattice constant of copper matrix that are non-monotonous versus the accumulated strain. The crystalline structure refinement upon the severe plastic deformation proceeds through selforganization of dense dislocation pile-ups into grain boundaries what implies a reverse influence of the second-phase particles, which are effective obstacles to dislocation motion, on the structural transformations. Thus, there exists an interdependence between strain mechanisms and phase transitions such as the second phase particles precipitation and their dissolution in the copper matrix that influences the structure refinement, the dispersion particles distribution in the bulk and, consequently, the strength and other properties of the material. This influence may be especially complicated in alloys that like the one under study have a second phase consisted of particles of many different compositions.
\end{abstract}

\section{Роль фазовых превращений в эволюции дисперсных частиц в хромовых бронзах при равноканальном угловом прессовании}

\author{
Фаизов И.А. ${ }^{1}$, Рааб Г.И. ${ }^{1}$, Фаизова С.Н. ${ }^{1 \dagger}$, Зарипов Н.Г. ${ }^{1}$, Аксенов Д.А. ${ }^{2}$ \\ †snfaiz@mail.ru
}

1Уфимский государственный авиационно-технологический университет, ул. К.Маркса, 12, г.Уфа, 450000, Россия
²Институт молекул и кристаллов УНЦ РАН, Пр.Октября, 71, г. Уфа, 450000, Россия

Образцы низколегированного дисперсионно-упрочняемого сплава системы Cu-Cr-Zr были обработаны с использованием сочетания нескольких методов интенсивной пластической деформации. Предварительно образцы были закалены с предплавильной температуры для создания состояния пересыщенного твердого раствора. Эволюция средних размеров и плотности распределения частиц различных химических составов в ходе эксперимента указывает на протекание в сплаве фазовых превращений даже на этапе холодной деформации. В эволюцию определенно вносит вклад распад твердого раствора из-за деформационно-индуцированного ускорения диффузии, но весь комплекс наблюдаемых результатов не может быть объяснен только этим относительно хорошо изученным явлением и требуют привлечения предположения о деформационно-индуцированном растворении частиц вторых фаз. В работе обсуждается возможный механизм растворения, связывающий этот процесс с механической фрагментацией частиц при интенсивной пластической деформации. Отмечается зависимость эффективности растворения от состава и определяемой им морфологии частиц. Вывод об одновременном протекании кинетически-разнонаправленных фазовых 
превращений подтверждается также наблюдением немонотонного изменения постоянной решетки медной матрицы при увеличении накопленной деформации. Измельчение структуры материала при обработке с использованием интенсивной пластической деформации происходит путем самоорганизации дислокационных скоплений в границы кристаллитов, что подразумевает обратное влияние ансамбля дисперсионно-упрочняющих частиц, являющихся препятствиями для движения дислокаций, на структурные превращения. Таким образом, имеет место взаимодействие деформационных механизмов и фазовых превращений типа выделения частиц вторых фаз и растворения их в медной матрице, которое влияет на измельчение структуры, характер распределения частиц и, как следствие, на прочность и другие свойства материала. Такое влияние может быть весьма сложным в сплавах, подобных изученному, где вторая фаза представлена частицами различных составов.

Ключевые слова: равноканальное угловое прессование (РКУП), медные сплавы, фазовые превращения, частицы.

\section{1. Введение}

Интенсивная пластическая деформация (ИПД) является одним из перспективных методов получения материалов с наноразмерной кристаллической структурой. Материалы после наноструктурирования демонстрируют сочетания функциональных свойств, отличные от тех, которые формируются при традиционных обработках [1-4]. Результатами многочисленных исследований показано, что наноструктурирование электропроводных низколегированных хромовых бронз позволяет существенно повысить механические характеристики этих сплавов при сохранении высокой электро- и теплопроводности [5-8].

Исследуемый сплав системы $\mathrm{Cu}-\mathrm{Cr}-\mathrm{Zr}$ относится к классу дисперсионно-упрочняемых сплавов, для которых морфология и характер распределения в матрице частиц вторых фаз играет существенную роль для создания в материале оптимального сочетания основных функциональных свойств - прочности, термостабильности и электропроводности [5-8].

В ходе ИПД частицы вторых фаз проходят сложную эволюцию, затрагивающую их размеры, морфологию и распределение в материале. Происходящие изменения вызываются как механической фрагментацией частиц, так и деформационно-стимулированными фазовыми превращениями - растворением и выделением частиц $[5,8-12]$. Благодаря большой плотности дислокаций и других структурных дефектов при ИПД происходит их пространственная самоорганизация, которая на микроуровне порождает значительные локальные неоднородности. Это обстоятельство позволяет допустить одновременное протекание кинетически разнонаправленных процессов переноса атомов между частицами вторых фаз и твердым раствором, которые скоррелированы с кристаллической и дефектной структурой материала. Вторые фазы в рассматриваемых бронзах являются препятствием для движения дислокаций, что создает обратное влияние фазовых превращений на деформирование и, как следствие, формирование наноструктуры.

Поскольку описанные явления определяются нетривиальным взаимодействием процессов разной природы, их экспериментальное изучение связано со значительными трудностями и требует целостного анализа широкого круга разнородных данных. В данной работе приводятся результаты исследования эволюции ансамбля частиц вторых фаз и сопутствующего изменения свойств материала в ходе ИПД и последующего пост-деформационного старения.

\section{2. Материал и методики исследования}

В состоянии поставки образцы сплава $\mathrm{Cu}-1 \mathrm{Cr}-0.7 \mathrm{Al}-0.2 \mathrm{Zr}$ имели форму прутков диаметром 40 мм после стандартной промышленной обработки. На первом этапе - при высокотемпературной обработке - образцы подвергались выдержке в течение 1 часа при температуре $1050^{\circ} \mathrm{C}$ с последующей закалкой в воду для создания твердого раствора легирующих элементов в медной матрице. Далее образцы подвергались холодной деформации равноканальным угловым прессованием (РКУП) с последующей кузнечной протяжкой (КП) и волочением (В). Пост-деформационное старение проводилось при температуре $450^{\circ} \mathrm{C}$ в течение 1 часа. РКУП проводили на оснастке с углом пересечения каналов $90^{\circ}$ по маршруту Вс при комнатной температуре, количество проходов РКУП -8 , значение накопленной деформации $-e=1.1 \cdot 8=8.8$ [1-3]. Обработка кузнечной протяжкой и последующее волочение проводились до сужений, соответственно, $56 \%$ и $25 \%$.

На всех этапах проводилось исследование структуры материала с помощью оптической металлографии (ОМ), растровой электронной микроскопии (РЭМ), в том числе и EBSD-анализа, просвечивающей электронной микроскопии (ПЭМ) фольг и экстракционных реплик.

Для детального исследования изменения размеров и характера распределения частиц в ходе РКУП использовали метод экстракционных реплик. С учетом специфических особенностей отделения частиц от матрицы, получаемые статистические данные о размерах частиц и расстоянии между ними могут иметь погрешности в области наибольших и наименьших наблюдаемых размеров. Однако учитывая большую статистику проведенных измерений, составившую не менее 1000 частиц для каждого состояния, характер изменения этих величин можно оценить с достаточной уверенностью. Размер частиц определялся как среднее от измерений в двух взаимно перпендикулярных направлениях. Среднее расстояние между частицами в плоскости рассчитывали по формуле:

$$
L=\sqrt{\frac{S}{N}},
$$

где $S$ - площадь поверхности образца, $N-$ количество частиц на площади $S$.

Исследование реплик в ПЭМ позволило при расшифровке электронограмм отдельных частиц установить, что частицы определенных составов имеют характерную морфологию. Этот факт был использован для дальней- 
шей идентификации частиц при получении статистических данных.

Механические испытания на растяжение проводили на универсальном динамометре «Instron» при комнатной температуре, скорости деформации $5,5 \times 10^{4} \mathrm{c}^{-1}$, измерения микротвердости по Виккерсу (при нагрузке $1 \mathrm{H}$ и с выдержкой 10 секунд) - на микротвердомере Micromet 5101

\section{3. Результаты и их обсуждение}

Средний размер зерен после высокотемпературной обработки сплава составляет $34 \pm 3$ мкм, наблюдается заметное количество частиц. Поскольку средние размеры частиц в этом состоянии довольно малы, их присутствие, по-видимому, связано с недостаточной скоростью закалки.

Обработка методами ИПД (РКУП+КП+В) приводит к существенному измельчению структуры - формируется вытянутая вдоль прутка полосовая структура (рис. 1 а). Поперечный размер фрагментов составляет примерно 200-240 нм.

Общая плотность частиц уменьшается приблизительно в два раза по сравнению с состоянием после высокотемпературной обработки. Хотя частицы наблюдаются как по границам, так и в теле зерен (рис. 1, 2), обращает на себя внимание корреляция их расположения с особенностями дефектной структуры и границами фрагментов. Большое количество мелких частиц наблюдается, как правило, в скоплениях дислокаций.
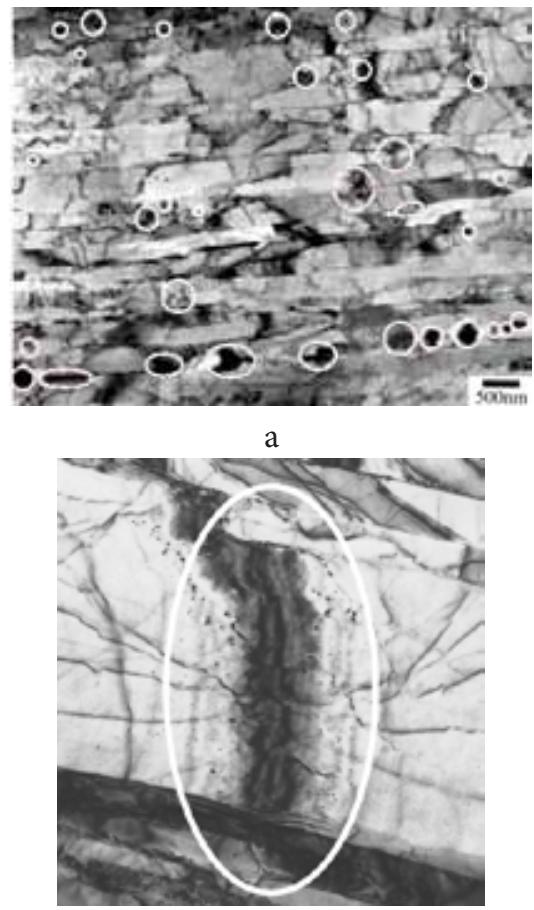

$\mathrm{b}$

Pис. 1. (а) Полосовая структура сплава $\mathrm{Cu}-1 \mathrm{Cr}-0.7 \mathrm{Al}-0.2 \mathrm{Zr}$ после комплексной ИПД. (b) Мелкие частицы хорошо видны в зерне вблизи полос экстинции.

Fig. 1. (a) Band structure of $\mathrm{Cu}-1 \mathrm{Cr}-0.7 \mathrm{Al}-0.2 \mathrm{Zr}$ alloy after the complex SPD. (b) Small particles are clearly visible in the grain close to the fringes of extinction.
На этапе пост-деформационного старения поперечный размер фрагментов не изменился, наблюдаемая плотность частиц вторых фаз увеличилась примерно в 4 раза по сравнению с состоянием после ИПД, причем значительно возрастает доля мелких, до 20 нм, частиц.

На каждом этапе обработки производилось определение статистических характеристик ансамблей частиц, различающихся по морфологическим признакам и химическому составу. В таблице 1 показано, как изменялся средний размер частиц различного состава на разных этапах обработки. Стрелками показано направление изменения среднего размера частиц.

Из таблицы 1 видно, что средние размеры частиц разного состава на этапе ИПД меняются разнонаправленно. После высокотемпературной обработки не наблюдаются присутствовавшие в состоянии поставки медно-алюминиевые частицы. Они вновь появляются после ИПД, причем их средний размер в этом состоянии даже больше, чем в состоянии поставки. Такое поведение однозначно свидетельствует о деформационно-стимулированном распаде твердого раствора в ходе ИПД.

В таблице 2 приведены средние расстояния между частицами, отражающие плотность их распределения, вычисленные по всему ансамблю частиц. Ввиду многообразия химического состава задача определения изменения числа частиц для каждой из компонент ансамбля выходит за рамки данной работы.

Наблюдаемая корреляция между частицами и элементами фрагментированной структуры материала может объясняться тем, что частицы, являясь эффективными стопорами дислокаций (рис. 2 b), накапливают вблизи себя плотные скопления, которые затем трансформируются в границы фрагментов. Альтернативный

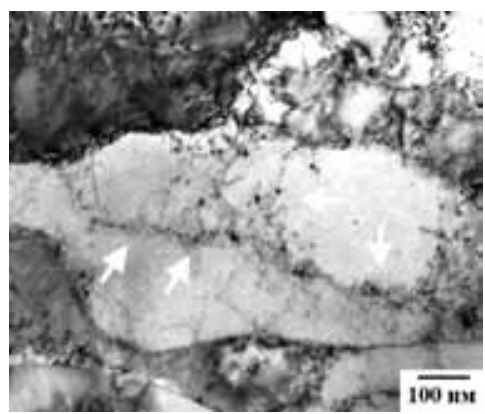

a

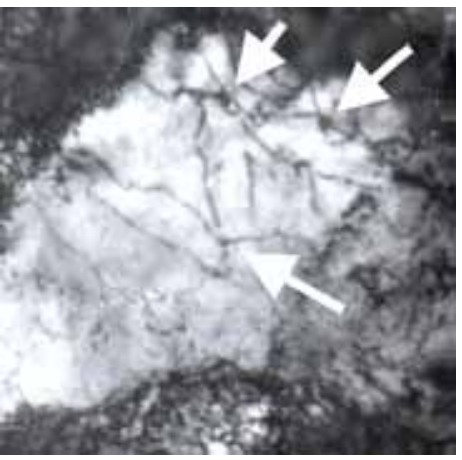

$\mathrm{b}$

Рис. 2. Взаимодействие частиц и дислокаций (a и b).

Fig. 2. Interaction between particles and dislocations $(a, b)$. 
Табл. 1. Изменение среднего размера (в нанометрах) частиц вторых фаз сплава $\mathrm{Cu}-1 \mathrm{Cr}-0.7 \mathrm{Al}-0.2 \mathrm{Zr}$ на разных этапах обработки. Каждый тип частиц характеризуется типичной морфологией, электронограммой и химическим составом.

Table 1. Changes of average sizes (in nanometers) of particles of second phases with processing stage in $\mathrm{Cu}-1 \mathrm{Cr}-0.7 \mathrm{Al}-0.2 \mathrm{Zr}$ alloy. Each particle type is characterized by its typical morphology, electron diffraction pattern and chemical composition.

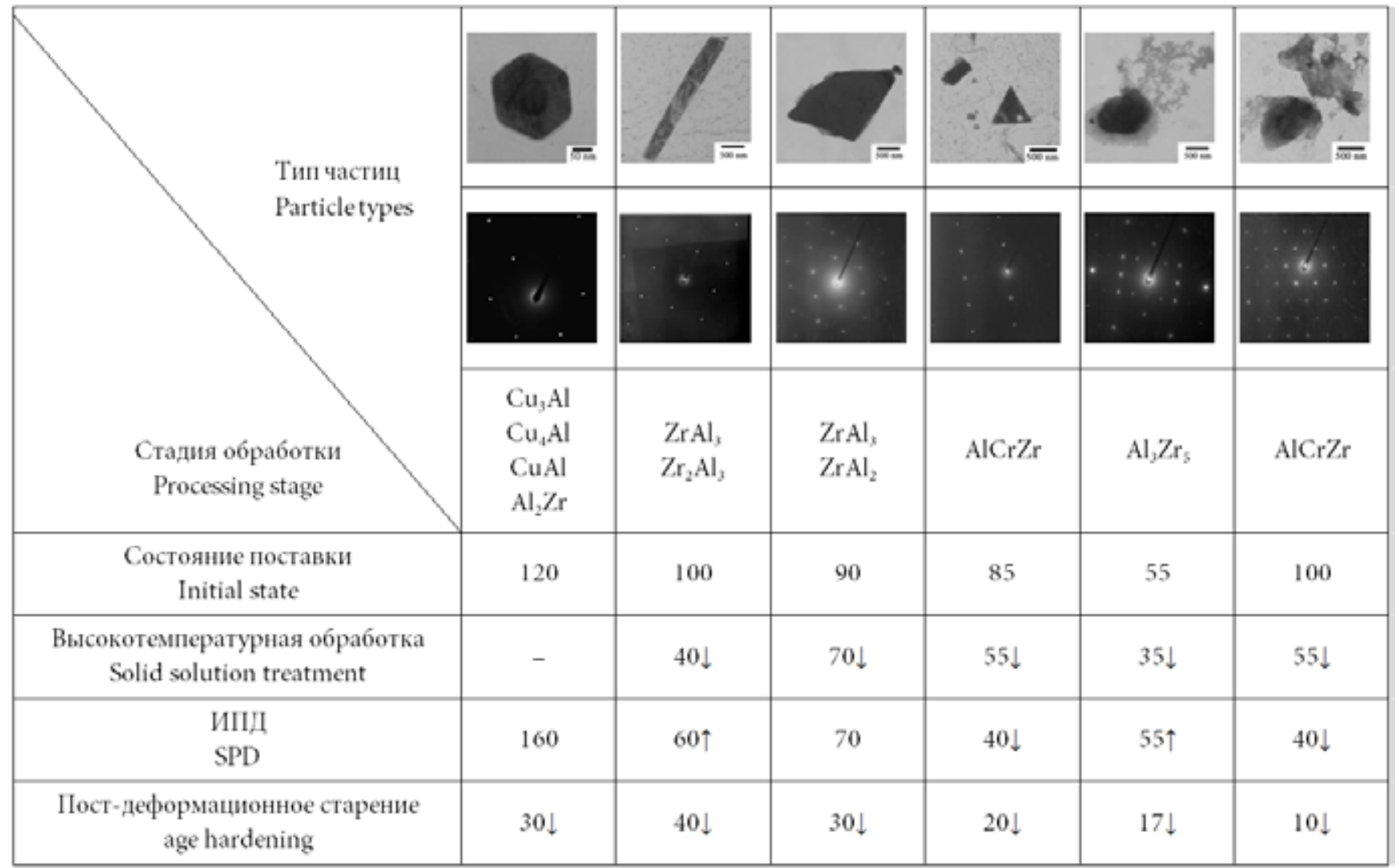

Табл. 2. Изменения среднего размера частиц и среднего расстояния между ними на различных этапах обработки. Table 2. Dependence of average particle size and average distance between particles on the processing stage.

\begin{tabular}{|c|c|c|c|c|}
\hline $\begin{array}{c}\text { Виды обработки } \\
\text { Processing stage }\end{array}$ & $\begin{array}{c}\text { Поставка } \\
\text { Initial }\end{array}$ & $\begin{array}{c}\text { Высокотемпературная } \\
\text { обработка } \\
\text { Solid solution treatment }\end{array}$ & $\begin{array}{c}\text { ИПд } \\
\text { SPD }\end{array}$ & $\begin{array}{c}\text { Пост-деформационное } \\
\text { старение } \\
\text { age hardening }\end{array}$ \\
\hline $\begin{array}{c}\text { Средний размер частиц, нм } \\
\text { The average particle size, nm }\end{array}$ & 80 & $40 \downarrow$ & $50 \uparrow$ & $20 \downarrow$ \\
\hline $\begin{array}{c}\text { Среднеe paсстояние между частицами, нм } \\
\text { The average distance between particles, nm }\end{array}$ & 960 & $460 \downarrow$ & $680 \uparrow$ & $360 \downarrow$ \\
\hline
\end{tabular}

механизм, учитывающий присутствие деформационно-индуцированного выделения частиц, может заключаться в том, что дислокационные скопления и границы фрагментов являются стоками точечных дефектов и, таким образом, предпочтительными местами зарождения новых частиц. По нашему мнению, наиболее вероятно одновременное синергетическое действие обоих механизмов.

В то же время ИПД вызывает значительное уменьшение общего числа частиц, что указывает на доминирование обратного процесса, а именно, деформационно-индуцированного растворения частиц, тесно связанного с их механической фрагментацией [12]. При данной обра- ботке механическая фрагментация частиц может происходить только путем квазихрупкого разлома, поскольку дислокационное перерезание при достигнутых значениях накопленной деформации не может играть заметной роли.

По своему составу частицы являются интерметаллидами, имеющими кристаллическую решетку, некогерентную с медью, вследствие чего граница раздела частица-матрица трудно проницаема для дислокаций. Являясь препятствием движению дислокаций, частица аккумулирует на себе скопление, создающее напряжения, достаточные для развития в ней внутренних сдвигов и, в итоге, для ее разрушения. 
Образовавшиеся фрагменты имеют участки с высокой кривизной поверхности (рёбра), что делает термодинамически возможным их частичное растворение. На протекание растворения влияет ускорение диффузии при ИПД [13]. Также в ускоренный массоперенос может давать вклад дрейф примесных атомов в поле дислокационных скоплений, релаксирующих после разрушения частицы. Эффективность данного процесса зависит от степени метастабильности конкретного интерметаллида, что объясняет сложный характер изменения размеров частиц разных составов. Вызванное процессом растворения уменьшение среднего размера частиц делает часть из них недоступными для обнаружения методом ПЭМ, что объясняет наблюдаемое уменьшение их количества после ИПД.

Еще одним свидетельством протекания при ИПД кинетически разнонаправленных процессов растворениявыделения может служить поведение параметра решетки медной матрицы, зависящего от концентрации твердого раствора легирующих элементов. В таблице 3 приведены данные РСА о значении параметра решетки после разного количества проходов РКУП, полной ИПД обработки и пост-деформационного старения. Видно, что изменение этой величины происходит немонотонно. Поскольку на этапе деформирования нет оснований ожидать немонотонности вклада от напряжений, создаваемых дефектной структурой, основной причиной такого поведения следует считать изменения концентрации твердого раствора в результате фазовых превращений.

Bсе эти изменения свидетельствуют о том, что в условиях ИПД происходят деформационно-стимулированный распад ТР и деформационно-индуцированное растворение частиц вторых фаз.

При пост-деформационном старении завершается распад твердого раствора и, соответственно, появляется большое количество мелких, 20 нм, частиц, которые и определяют окончательно достигнутую высокую прочность в данном сплаве - $700 \mathrm{MПа} \mathrm{(табл.} \mathrm{4).} \mathrm{Для} \mathrm{сравне-}$ ния, после промышленной обработки средний размер частиц и среднее расстояние между ними составляют 80 и 960 нм, соответственно, а прочность равна $550 \mathrm{MПа.}$

Высокие прочностные характеристики после комплексной обработки сплава возможны лишь благодаря взаимодействию двух основных механизмов упрочнения - структурного и дисперсионного. Как показывают приведенные в работе результаты, во взаимодействии этих механизмов важную роль играют фазовые превращения, наблюдаемые в условиях ИПД.

Табл. 3. Параметр решетки меди в сплаве $\mathrm{Cu}-1 \mathrm{Cr}-0.7 \mathrm{Al}-0.2 \mathrm{Zr}$ на различных каждом этапах обработки.

Table 3. The copper lattice constant of alloy $\mathrm{Cu}-1 \mathrm{Cr}-0.7 \mathrm{Al}-0.2 \mathrm{Zr}$ on different stages of treatment.

\begin{tabular}{|c|c|c|c|c|c|c|}
\hline $\begin{array}{c}\text { Стадия обработки } \\
\text { Treatment stage }\end{array}$ & $\begin{array}{c}\text { Высокотемпературная } \\
\text { обработка }\end{array}$ & $\begin{array}{c}\text { РКУП } \\
\text { S проходов } \\
\text { ECAP }\end{array}$ & $\begin{array}{c}\text { РКУП } \\
8 \text { проходов } \\
\text { ECAP } \\
8 \text { passes }\end{array}$ & $\begin{array}{c}\text { ИПд } \\
\text { SPD }\end{array}$ & $\begin{array}{c}\text { Старение } \\
\left(450^{\circ} \mathrm{C}\right) \\
\text { age hardening } \\
\left(450^{\circ} \mathrm{C}\right)\end{array}$ & $\begin{array}{c}\text { Табличное } \\
\text { 3начение, } \AA \\
\text { Standard value, } \AA\end{array}$ \\
\hline Параметр & $3.617(7) \pm 0.0002$ & $\begin{array}{c}3.614(2) \pm \\
0.0001 \downarrow\end{array}$ & $\begin{array}{c}3.616(1) \pm \\
0.0001 \uparrow\end{array}$ & $\begin{array}{c}3.617(2) \pm \\
0.0002\end{array}$ & $\begin{array}{c}3.614(5) \pm \\
0.0003 \downarrow\end{array}$ & 3.615 \\
решетки Cu, $\AA$ & & & & \\
\hline
\end{tabular}

Табл. 4. Механические свойства сплава на различных этапах обработки.

Table 4. Mechanical properties of alloys on different stages of processing.

\begin{tabular}{|c|c|c|}
\hline $\begin{array}{c}\text { Стадия обработки } \\
\text { Processing stage }\end{array}$ & $\begin{array}{l}\text { Микротвердость, МПа } \\
\text { Microhardness, MPa }\end{array}$ & $\begin{array}{l}\text { Прочность } \sigma, \text { МПа } \\
\text { Tensile strength, MРa }\end{array}$ \\
\hline $\begin{array}{c}\text { Состояние поставки (после промышленной обработки) } \\
\text { Initial stage (after industrial processing) }\end{array}$ & 1450 & 550 \\
\hline $\begin{array}{l}\text { закалка с } 1050^{\circ} \mathrm{C} \\
\text { Solid solution treatment }\end{array}$ & 660 & 220 \\
\hline $\begin{array}{l}\text { ИПД (РКУП+К+В) } \\
\text { SPD }\end{array}$ & 1400 & 550 \\
\hline $\begin{array}{l}\text { старение }-450^{\circ} \mathrm{C} 1 \text { час } \\
\text { age hardening } 450^{\circ} \mathrm{C} 1 \mathrm{~h}\end{array}$ & 1980 & 700 \\
\hline
\end{tabular}




\section{4. Выводы}

Полученные экспериментальные результаты указывают на то, что в условиях равноканального углового прессования хромоциркониевой бронзы происходит не только измельчение структуры матрицы, но и сложные фазовые превращения, затрагивающие размеры и характер распределения частиц вторых фаз.

Анализ всей совокупности экспериментальных результатов, полученных на разных структурных уровнях, позволяет предположить, что одновременно реализуются два разнонаправленных процесса: деформационно-стимулированный распад твердого раствора легирующих элементов и деформационно-индуцированное растворение частиц вторых фаз.

Благодарность/Acknowledgements. Изготовление образиов методом РКУП и исследование механических свойств выполнено за счет гранта Российского научного бонда (проект № 14-19-01062) в ФГБОУ ВПО «Убимский государственный авиаиионный технический университет», изучение структурных и фазовых преврашений за счет проекта РФФИ № 14-08-97058 р_Поволжье_а.

\section{Литература/References}

1. V. M. Segal. Mater. Sci. Eng. 1995. V. A197. P. 157 - 164.

2. V.M. Segal, V.I. Reznikov, V.I. Kopylov etc. Processing of structure formation in metals. Minsk: Since and Technology, 1994. 231 p. (in Russian) [Сегал В.M., Резников В.И., Копылов В.И. и др. Процессы структурообразования металлов. Минск: Наука и техника, 1994. 231 c.]

3. R.Z. Valiev, I. V. Aleksandrov. Nanostructured materials obtained by severe plastic deformation. M.: Logos, 2000. 272 p. (in Russian) [Валиев Р. 3., Александров И. В. На ноструктурные материалы полученные интенсивной пластической деформацией. М.: Логос, 2000. 272 с.]

4. R.A. Andrievskiy, A.M. Glezer. Uspekhi fi zicheskikh nauk, 2009, T. 179, № 4, p. 337 - 358. DOI: $10.3367 / \mathrm{UF}$ Nr.0179.200904a.0337 (in Russian) [Андриевский Р. А., Глезер А. М. Прочность наноструктур. УФН, 2009, Т. 179$, c. $337-358]$

5. S. Faizova, G. Raab, D. Aksenov, N. Zaripov, I. Faizov/The physical aspects of the formation of highstate precipitation hardened alloys under severe plastic deformation by torsion // Physical Mesomechanics. № 4 . 2015 [С. Н. Фаизова (4), Г.И. Рааб (19), Д. А. Аксенов (0), И.А. Фаизов (0)/Физические аспекты формирования высокопрочного состояния дисперсионно-упрочняемых сплавов при интенсивной пластической деформации кручением // «Физическая мезомеханика», № 4, 2015]

6. A. Vinogradov, V. Patlan, Y. Suzuki, K. Kitagawa, V. Kopylov. Acta Materialia. 2002. V.50, P.1639-1651. DOI: 10.1016/S1359-6454 (01) 00437-2
7. A. Vinogradov, T. Ishida ets. Acta Materialia. 2005. V.53. P.2181 - 2192. DOI: 10.1016/j.actamat.2005.01.046

8. S. N. Faizova, R.Z. Valiev, N.V. Mazhitova, G.I. Raab. The kinetics of non-equilibrium solid solution of $\mathrm{Cu}-\mathrm{Cr}$ during intensive plastic deformation by torsion. Electronic journal «Phase transitions, ordered States and new materials». 4. 2010. URL: http://ptosnm.ru/ru/issue/2010/4/49/publication/533(inRussian) [Фаизова С.Н., Валиев Р.3., Мажитова Н.В., Рааб Г.И.. Неравновеснвя кинетика твердого раствора системы $\mathrm{Cu}-\mathrm{Cr}$ при интенсивной пластической деформации кручением. Электронный журнал «Фазовые переходы, упорядоченные состояния и новые материалы». 4. 2010. URL: http://ptosnm.ru/ru/issue/2010/4/49/publication/533]

9. V. V. Sagaradze, V. A. Shabashov, T. M. Lapina, et al. Lowtemperature strain-induced dissolution of intermetallic $\mathrm{Ni}^{3} \mathrm{Al}(\mathrm{Ti}, \mathrm{Si}, \mathrm{Zr}$ ) phases in $\mathrm{Fe}-\mathrm{Ni}$ alloys with fcc lattice. Fiz. Met. Metalloved., 78 (6), 1994, 49-61. (in Russian) [B.В. Сагарадзе, В.А. Шабашов, Т. М. Лапина. Низкотемпературное деформационное растворение интерметаллидных фаз $\mathrm{Ni}^{3} \mathrm{Al}(\mathrm{Ti}, \mathrm{Si}, \mathrm{Zr})$ в Fe-Ni сплавах с ГЦК решёткой. ФММ. - 1994. - Т.78, N 6. C. $49-61]$

10. V.V. Sagaradze, S. V. Morozov, V.A. Shabashov, L. N. Romashev, I. R. Kuznetsov. Dissolution of spherical and lamellar intermetallics in Fe-Ni-Ti austenitic alloys during cold plastic deformation. Fiz. Met. Metalloved. 66 (2), 1988, Р. 328 - 338. (in Russian) [В. В. Сагарадзе, С.В. Морозов, В.А. Шабашов, Л.Н. Ромашёв, Р.И. Кузнецов. Растворение сферических и пластинчатых интерметаллидов в $\mathrm{Fe}-\mathrm{Ni}-\mathrm{Ti}$ аустенитных сплавах при холодной пластической деформации. ФММ. - 1988. - T.66, N 2. - C.328 - 338.]

11. V.A. Shabashov Nonequilibrium diffusion phase transformations and nanostructuring in intense cold deformation. Materials science. 2008. N 3 (55). P. 169 - 179 (in Russian) [Шабашов, В. А. Неравновесные диффузионные фазовые превращения и наноструктурирование при интенсивной холодной деформации. Вопросы материаловедения. - 2008. - N 3 (55). - C.169-179]

12. V.V. Sagaradze, Diffusion transformation in steels due to cold deformation, Metallovedenie i Termicheskaya Obrabotka Metallov, No. 9, pp. 19-27, September, 2008. (in Russian) [В.В. Сагарадзе Диффузионные превращения с сталях при холодной деформации, МиТОМ, 2008, № 9, c.19-27.]

13. Y.R. Kolobov, R.Z. Valiev. Grain-boundary diffusion and properties of nanostructure materials // Novosibirsk: Nauka, 2001, p. 232. (in Russian) [Колобов Ю.P., Валиев Р. 3 и др. Зернограничная диффузия и свойства наноструктурных материалов. Новосибирск: Наука, 2001, с. 232.] 